\title{
Performance of Space-Code-Division Multiple-Access Systems Using Reduced-Rank Detection
}

\author{
Jiliang Zhang and Lie-Liang Yang \\ School of ECS, University of Southampton, SO17 1BJ, UK \\ Tel: +44-23-8059 3364; Fax: +44-23-8059 4508 \\ E-mail: 1ly@ecs.soton.ac.uk; http://www-mobile.ecs.soton.ac.uk
}

\begin{abstract}
In this contribution the performance of multipleinput multiple-output space-division, code-division multipleaccess (MIMO SCDMA) is investigated, when the low-complexity reduced-rank detection is employed. In [1] a MIMO SCDMA system is proposed, aiming at improving the capacity of the existing direct-sequence (DS) CDMA (DS-CDMA) systems. The performance of the SCDMA system in [1] is investigated, when the fullrank multiuser detectors (MUDs) are considered. However, since the rank of the detection-related matrices in SCDMA is usually high, the complexity is extremely high, even linear detectors, such as decorrelating, minimum mean-square error (MMSE), etc. are employed. In this paper the reduced-rank detection scheme, which is derived based on the Taylor polynomial approximation (TPA), is proposed for detecting the SCDMA signal. The bit error rate (BER) performance of the MIMO SCDMA is investigated, when communicating over frequency-selective fading channels. It can be shown that the considered reduced-rank detection is capable of achieving a BER performance that is close to that of using fullrank detection, but with significantly lower detection complexity.
\end{abstract}

\section{INTRODUCTION}

In wireless communications DS-CDMA has been a typical multiple-access scheme in the second and third generations of wireless communications systems. Without any doubt, it will constitute an important candidate in the future generations of wireless communications systems. Hence, one of the important issues in future wireless communications is to increase the capacity of DS-CDMA networks. In [1] a MIMO SCDMA scheme is proposed and investigated, where multiple transmit and receive antennas are introduced in order to enhance the capacity and performance of the DS-CDMA systems. It can be shown that the MIMO SCDMA assisted by MUD constitutes a highly promising multiple-access scheme, which is capable of efficiently exploiting the system resources and significantly increasing the capacity of a conventional DS-CDMA system.

In [1] the performance of the MIMO SCDMA system has been investigated in the context of a range of full-rank linear MUDs, including decorrelating and MMSE [2]. However, in the MIMO SCDMA systems the rank of the detection-related matrices is contributed by both the DS-CDMA's code signatures as well as the SDMA's spatial signatures. Hence, the rank in detection is usually very high, yielding high-complexity detection, even when the above-mentioned linear MUDs are employed. In order to mitigate the detection complexity in the SCDMA system [1], therefore, in this contribution reducedrank detection is proposed and investigated. Specifically, the reduced-rank MMSE detection is investigated in the context of the SCDMA, where the reduced-rank subspace is derived based on the principles of Taylor polynomial approximation (TPA) [3]. The BER performance of the SCDMA system using the proposed reduced-rank MMSE detection is investigated, when communicating over multipath fading channels. Our study and simulation results show that the TPA-assisted reduced-rank detection is capable of achieving a BER performance that is close to that of using full-rank detection, but with significantly lower detection complexity.

\section{System Description}

In this section we provide an overview of the MIMO SCDMA system proposed in [1], as well as provide the required MIMO equations for carrying out the further discussion and analysis.

\section{A. Transmitted Signal}

The MIMO system considered in this paper is the same as that in [1], which consists of $U$ transmit antennas and $V$ receive antennas. The transmitter schematic diagram of the $k$ th user is shown in Fig.1, where real-valued data symbols using BPSK baseband modulation and real-valued spreading [4] were assumed. As shown in Fig.1, at the transmitter side the binary input data stream having a bit duration of $T_{b}$ is serial-to-parallel (S-P) converted to $U$ parallel sub-streams. The new bit duration of each parallel sub-stream or the symbol duration becomes $T_{s}=U T_{b}$. After S-P conversion, the $U$ parallel bits are directsequence spread using the STS schemes proposed in [4] with the aid of $U$ pseudo-noise (PN) spreading sequences having a period of $U N$, where $N=T_{b} / T_{c}$ represents the number of chips per bit time-duration and $T_{c}$ is the chip-duration of the PN spreading sequence. As seen in Fig.1, following STS, the $U$ parallel signals are mapped to the $U$ transmit antennas, where the $U$ parallel signals are carrier modulated and transmitted by the corresponding $U$ antennas. 


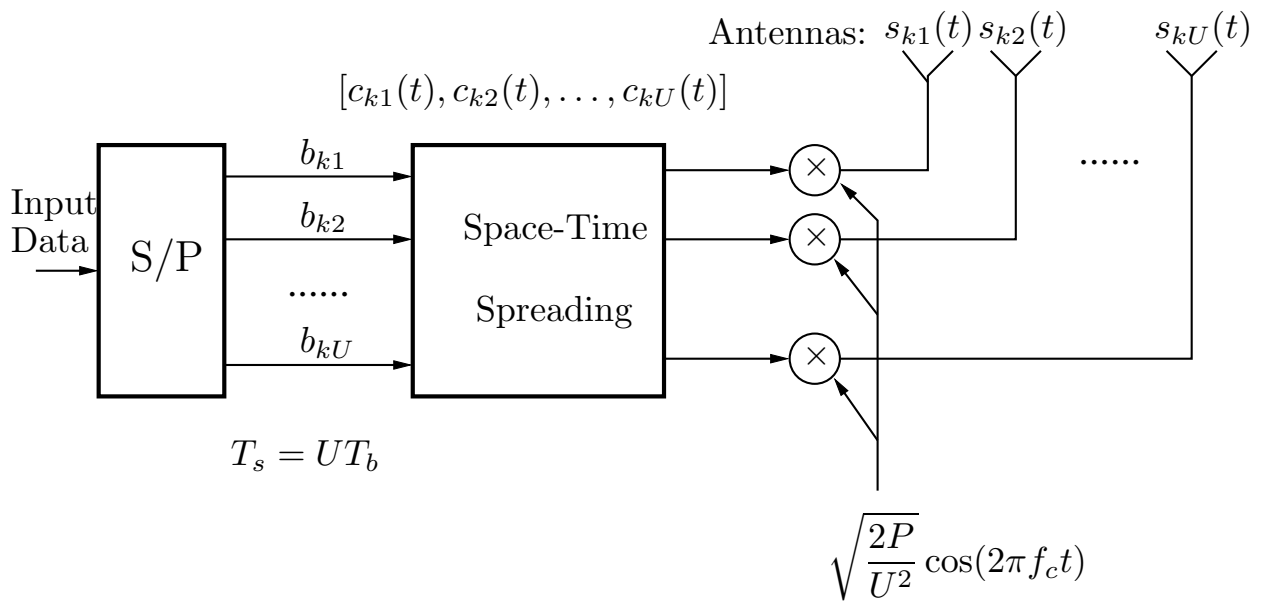

Fig. 1. Transmitter block diagram of the MIMO SCDMA system using space-time spreading (c)IEEE, [1].

As shown in [1], for the case of using $U=2$ transmit antennas, the transmitted signal of the $k$ th user within a data block of $M$ symbol durations can be expressed as

$\boldsymbol{s}_{k}(t)=\sum_{m=0}^{M-1}\left[\begin{array}{l}\sqrt{\frac{2 P}{2^{2}}}\left[b_{k 1}(m) c_{k 1}\left(t-m T_{s}\right)+b_{k 2}(m)\right. \\ \left.\times c_{k 2}\left(t-m T_{s}\right)\right] \cos \left(2 \pi f_{c} t+\phi_{k 1}\right) \\ \sqrt{\frac{2 P}{2^{2}}}\left[b_{k 2}(m) c_{k 1}\left(t-m T_{s}\right)-b_{k 1}(m)\right. \\ \left.\times c_{k 2}\left(t-m T_{s}\right)\right] \cos \left(2 \pi f_{c} t+\phi_{k 2}\right)\end{array}\right]$

where $P$ represents each user's transmitted power, which is constant for all users, $U \times U=2^{2}$ is a power normalization factor, where the first $U$ is due to the $U$ number of transmit antennas, while the second $U$ is due to the symbol duration of $T_{s}=U T_{b}$. In (1) $s_{k}(t)=\left[s_{k 1}(t) s_{k 2}(t)\right]^{T}$ - where $T$ denotes vector or matrix transpose - represents the transmitted signal vector of the $U=2$ transmit antennas, while $M$ represents the number of symbols transmitted per block. As shown in (1), for the case of $U=2$, the $m$ th symbol contains two bits of $b_{k 1}(m)$ and $b_{k 2}(m)$, which are transmitted with the aid of the STS scheme based on two transmit antennas [4]. The STS sequences are assumed the periodic PN sequences having a period of $T_{s}=U T_{b}=U N T_{c}$, which can be expressed in the form of $c_{k u}(t)=\sum_{j=0}^{U N-1} c_{k u}^{j} \psi_{T_{c}}\left(t-j T_{c}\right), u=1,2, \ldots, U$, where $c_{k u}^{j}$ assumes values of +1 or -1 , while $\psi_{T_{c}}(t)$ is the chip waveform, which is defined over the interval $\left[0, T_{c}\right)$ and has the property of $\int_{0}^{T_{c}} \psi_{T_{c}}^{2}(t) d t=T_{c}$. Finally, in (1) $f_{c}$ represents the carrier frequency and $\phi_{k u}, u=1,2$ represents the initial phase with respect to the $u$ th antenna of the $k$ th user.

We assume that the SCDMA signals are transmitted over frequency-selective fading channels, where signal transmitted from any transmit antenna to any receive antenna experiences independent frequency-selective Rayleigh fading. The channel is assumed to be time-invariant over a data block time-duration, while experiences independent fading for different data blocks. Consequently, for a given data block, the complex low-pass equivalent representation of the CIR corresponding to the $u$ th transmit antenna of user $k$ and the $v$ th receive antenna is given by [5]

$$
h_{v u}^{(k)}(t)=\sum_{l=0}^{L-1} h_{v u}^{(k)}(l) \delta\left(t-l T_{c}\right)
$$

where $h_{v u}^{(k)}(l)=\left|h_{v u}^{(k)}(l)\right| \exp \left(j \phi_{v u}^{(k)}(l)\right)$ and $\tau_{k l}=l T_{c}$ represent the attenuation factor and delay of the $l$ th multipath component, respectively, while $L$ is the total number of resolvable multipath components and $\delta(t)$ is the Kronecker Delta-function. We assume that the phases $\left\{\phi_{v u}^{(k)}(l)\right\}$ in (2) are independent identically distributed (iid) random variables uniformly distributed in the interval $[0,2 \pi)$, while the $L$ multipath attenuations $\left\{\left|h_{v u}^{(k)}(l)\right|\right\}$ are independent Rayleigh random variables with a Probability Density Function (PDF) of [5]

$$
p_{\left|h_{v u}^{(k)}(l)\right|}(R)=\frac{2 R}{\Omega} e^{-R^{2} / \Omega}, R>0
$$

where $\Omega=E\left[\left|h_{v u}^{(k)}(l)\right|^{2}\right]$ is the second moment of $h_{v u}^{(k)}(l)$.

The receiver structure for detection of the MIMO SCDMA signal is shown in Fig.2. The receiver comprises of $V$ receive antennas. At each of the $V$ receive antennas, the received signal is first passed through a filter matched to the transmitted chipwaveform of $\psi_{T_{c}}(t)$. Then, the output of the matched-filters are sampled at a rate of $1 / T_{c}$. Hence, in a general MIMO SCDMA system using $U$ transmit antennas, each receive antenna branch provides $(M U N+L-1)$ samples corresponding to a data block, where $(L-1)$ is the result of channel delay, and the detector can collect a total of $V \times(M U N+L-1)$ samples from the $V$ receive antennas. As shown in Fig. 2 the antenna output samples are sent to a detector, where the data transmitted by the $K$ users is detected based on the TPA-assisted reduced-rank detection algorithms, which will be investigated in detail in our forthcoming discourse.

Let

$$
\boldsymbol{y}[m]=\left[\boldsymbol{y}_{1}[m]^{T}, \boldsymbol{y}_{2}[m]^{T}, \ldots, \boldsymbol{y}_{V}[m]^{T}\right]^{T}
$$




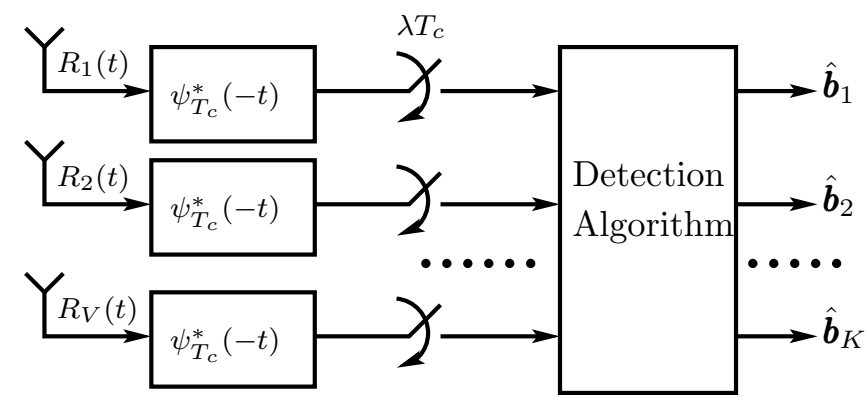

Fig. 2. Receiver schematic block diagram of the MIMO SCDMA systems (C)IEEE, [1].

represents the collection of all the samples from the $V$ receive antennas, which is related to the STS symbols transmitted within the $m$ th symbol duration by the $K$ users, and $\boldsymbol{y}[m]$ is a $(U N+L-1) V$-length vector. Then, upon following the analysis in [1], it can be shown that $\boldsymbol{y}[m]$ can be expressed as

$$
\begin{aligned}
\boldsymbol{y}[m]= & \sum_{k=1}^{K}\left\{\left(\boldsymbol{I}_{V} \otimes \boldsymbol{C}_{k}[0]\right) \boldsymbol{H}_{k} \boldsymbol{b}_{k}[m]\right. \\
& +\left(\boldsymbol{I}_{V} \otimes \underline{\boldsymbol{C}}_{k}[-1]\right) \boldsymbol{H}_{k} \boldsymbol{b}_{k}[m-1] \\
& \left.+\left(\boldsymbol{I}_{V} \otimes \overline{\boldsymbol{C}}_{k}[1]\right) \boldsymbol{H}_{k} \boldsymbol{b}_{k}[m+1]\right\}+\boldsymbol{n}[m]
\end{aligned}
$$

where $\otimes$ represents the Kronecker product [6] operation, $\boldsymbol{n}[m]$ is a $(U N+L-1) V$-length noise vector, which has zero-mean and a covariance matrix of $E\left[\boldsymbol{n}[\mathrm{m}] \boldsymbol{n}[\mathrm{m}]^{H}\right]=$ $\left(U^{2} N_{0} / E_{c}\right) \boldsymbol{I}_{(U N+L-1) V}$, where $E_{c}$ represents the energy per chip. The other arguments in (5) are described as follows.

- $\boldsymbol{C}_{k}[0]: \mathrm{A}((U N+L-1) \times U L)$-dimensional matrix containing the $U$ STS sequences of user $k$ by taking into account of the $L$ multipath components. $\boldsymbol{C}_{k}[0]$ is corresponding to the current STS symbol received from user $k$;

- $\underline{\boldsymbol{C}}_{k}[-1]: \mathrm{A}((U N+L-1) \times U L)$-dimensional matrix due to the previous STS symbol of user $k$, but falling within its current symbol observation duration. Due to $\underline{C}_{k}[-1]$, the current STS symbol of user $k$ conflicts interference from the previous STS symbols of the $K$ users in the system;

- $\overline{\boldsymbol{C}}_{k}[1]: \mathrm{A}((U N+L-1) \times U L)$-dimensional matrix due to the following STS symbol of user $k$, but falling within its current symbol observation duration. Due to $\bar{C}_{k}[1]$, the current STS symbol of user $k$ conflicts interference from the following STS symbols of the whole $K$ users;

- $H_{k}:$ A $U L V \times U$ matrix corresponding to the CIRs connecting the $k$ th user to the $V$ receive antennas;

- $\boldsymbol{b}_{k}(m-1), \boldsymbol{b}_{k}(m), \boldsymbol{b}_{k}(m+1)$ : The $(m-1)$ th, $m$ th and $(m+1)$ th STS symbols transmitted by the $k$ th user, respectively.
Additionally, let

$$
\begin{aligned}
\boldsymbol{C}[0] & =\left[\left(\boldsymbol{I}_{V} \otimes \boldsymbol{C}_{1}[0]\right), \ldots,\left(\boldsymbol{I}_{V} \otimes \boldsymbol{C}_{K}[0]\right)\right] \\
\underline{\boldsymbol{C}}[-1] & =\left[\left(\boldsymbol{I}_{V} \otimes \underline{\boldsymbol{C}}_{1}[-1]\right), \ldots,\left(\boldsymbol{I}_{V} \otimes \underline{\boldsymbol{C}}_{K}[-1]\right)\right] \\
\overline{\boldsymbol{C}}[1] & =\left[\left(\boldsymbol{I}_{V} \otimes \overline{\boldsymbol{C}}_{1}[1]\right), \ldots,\left(\boldsymbol{I}_{V} \otimes \overline{\boldsymbol{C}}_{K}[1]\right)\right] \\
\boldsymbol{H} & =\operatorname{diag}\left\{\boldsymbol{H}_{1}, \boldsymbol{H}_{2}, \ldots, \boldsymbol{H}_{K}\right\} \\
\boldsymbol{b}[m] & =\left[\boldsymbol{b}_{1}^{T}[m], \ldots, \boldsymbol{b}_{K}^{T}[m]\right]^{T}
\end{aligned}
$$

Then, (5) can also be written as

$$
\begin{array}{r}
\boldsymbol{y}[m]=\boldsymbol{C}[0] \boldsymbol{H} \boldsymbol{b}[m]+\underline{\boldsymbol{C}}[-1] \boldsymbol{H} \boldsymbol{b}[m-1] \\
+\overline{\boldsymbol{C}}[1] \boldsymbol{H} \boldsymbol{b}[m+1]+\boldsymbol{n}[m]
\end{array}
$$

for $m=1,2, \ldots, M-2$. Note that, for $m=0$, the second component related to $\underline{C}[-1]$ on the right-hand side of (6) should be removed. By contrast, for $m=M-1$ the third component containing $\bar{C}[1]$ on the right-hand side of (6) should be removed. Let us now consider the reduced-rank detection of the STS assisted MIMO SCDMA signals.

\section{Linear Reduced-RAnK Detection of MIMO SCDMA SIGNALS}

In this section we focus on the linear reduced-rank detection of the MIMO SCDMA signals. Specifically, the reduced-rank detection based on the principles of TPA and MMSE [3] is investigated. This is because, in comparison with the other reduced-rank MMSE detectors, the TPA assisted reduced-rank MMSE detection does not depend on the eigen-decomposition of the auto-correlation matrix of the observation vector [7]. Furthermore, according to [7]-[9], the performance of TPAassisted reduced-rank MMSE detector is capable of converging to the full-rank performance with a rank significantly lower than that of the other types of reduced-rank MMSE detectors.

Let the output of a linear detector be expressed by

$$
\boldsymbol{z}[m]=\left[\boldsymbol{z}_{1}^{T}[m], \boldsymbol{z}_{2}^{T}[m], \ldots, \boldsymbol{z}_{K}^{T}[m]\right]^{T}
$$

which consists of the decision variables for the data-bits transmitted by the $K$ users during the $m$ th symbol duration, while $\boldsymbol{z}_{k}[\mathrm{~m}]$ contains the decision variables for $\boldsymbol{b}_{k}[\mathrm{~m}]$.

Let the rank in the detection subspace be $\mathcal{U}=\lambda U$. Then, according to [3], [8], [9], the detection subspace under TPA for the $k$ th user can be formed as

$$
\boldsymbol{P}_{\mathcal{U}}=\left[\boldsymbol{H}_{k}, \boldsymbol{R}_{y}^{(k)} \boldsymbol{H}_{k}, \ldots,\left(\boldsymbol{R}_{y}^{(k)}\right)^{\lambda-1} \boldsymbol{H}_{k}\right]
$$

where, by definition, $\boldsymbol{H}_{k}=\left(\boldsymbol{I}_{V} \otimes \boldsymbol{C}_{k}[0]\right) \boldsymbol{H}_{k}$, which is given in (5), $\boldsymbol{R}_{y}^{(k)}$ is the auto-correlation matrix of $\boldsymbol{y}[\mathrm{m}]$ of (5), which can be expressed as

$$
\begin{array}{r}
\boldsymbol{R}_{y}^{(k)}=\sum_{k=1}^{K}\left\{\left(\boldsymbol{I}_{V} \otimes \boldsymbol{C}_{k}[0]\right) \boldsymbol{H}_{k} \boldsymbol{H}_{k}^{H}\left(\boldsymbol{I}_{V} \otimes \boldsymbol{C}_{k}^{T}[0]\right)\right. \\
+\left(\boldsymbol{I}_{V} \otimes \underline{\boldsymbol{C}}_{k}[-1]\right) \boldsymbol{H}_{k} \boldsymbol{H}_{k}^{H}\left(\boldsymbol{I}_{V} \otimes \underline{\boldsymbol{C}}_{k}^{T}[-1]\right) \\
\left.+\left(\boldsymbol{I}_{V} \otimes \overline{\boldsymbol{C}}_{k}[1]\right) \boldsymbol{H}_{k} \boldsymbol{H}_{k}^{H}\left(\boldsymbol{I}_{V} \otimes \overline{\boldsymbol{C}}_{k}^{T}[1]\right)\right\} \\
+\frac{U^{2} N_{0}}{E_{c}} \boldsymbol{I}_{(U N+L-1) V}
\end{array}
$$


Having obtained the detection subspace $P_{\mathcal{U}}$, the observation vector in (5) can be mapped into a subspace of $\mathcal{U}$ dimension, generating the observation vector of

$$
\overline{\boldsymbol{y}}=\underbrace{\left(\boldsymbol{P}_{\mathcal{U}}^{H} \boldsymbol{P}_{\mathcal{U}}\right)^{-1} \boldsymbol{P}_{\mathcal{U}}^{H}}_{\left(\boldsymbol{S}_{\mathcal{U}}^{(k)}\right)^{H}} \boldsymbol{y}
$$

where $\left(\boldsymbol{P}_{\mathcal{U}}^{H} \boldsymbol{P}_{\mathcal{U}}\right)^{-1}$ represents a normalization factor. Finally, in the subspace $\bar{y}$ is input to a MMSE detector, where it is processed by a MMSE filter and output estimates for the transmitted space-time symbols $\left\{\boldsymbol{b}_{k}[m]\right\}$, which can be expressed as

$$
\hat{\boldsymbol{x}}_{1}=\overline{\boldsymbol{W}}_{o}^{H} \overline{\boldsymbol{y}}
$$

where the weight matrix is given by

$$
\overline{\boldsymbol{W}}_{o}=\left[\left(\boldsymbol{S}_{U}^{(k)}\right)^{H} \boldsymbol{R}_{y}^{(k)} \boldsymbol{S}_{U}^{(k)}\right]^{-1}\left(\boldsymbol{S}_{U}^{(k)}\right)^{H} \mathcal{H}_{k}
$$

Let us now show some performance results in the next section.

\section{Performance Examples}

In this section we provide a range of simulation results, in order to illustrate the achievable performance of the MIMO SCDMA system in conjunction with the proposed reducedrank detection scheme, when communicating over frequencyselective Rayleigh fading channels. In our simulation examples, we assumed that the Gold sequences having a period of $N=31$ were the basic sequences assigned to the users. For each given user, the $U N$-length STS sequences were formed from its basic sequence. Specifically, in our simulation examples these $U N$-length STS sequences of a given user were obtained by concatenating the assigned basic $N$-length Gold sequence with the $U$-length Walsh Hadamard codes. Let $\boldsymbol{c}_{k}$ be the basic Gold sequence assigned to the $k$ th user. Then, for $U=2$ and 4 , the STS sequences of the $k$ th user were obtained as

$$
\left[\begin{array}{cc}
+1 & +1 \\
+1 & -1
\end{array}\right] \otimes \boldsymbol{c}_{k}, \quad\left[\begin{array}{cccc}
+1 & +1 & +1 & +1 \\
+1 & +1 & -1 & -1 \\
+1 & -1 & +1 & -1 \\
+1 & -1 & -1 & +1
\end{array}\right] \otimes \boldsymbol{c}_{k}
$$

Furthermore, in our simulations we assumed a frequencyselective channel associated with $L=4$ multipath components. Note that, in our simulation results shown in Figs. $3-6$, as defined, $\lambda=\mathcal{U} / U$. Hence, for given values of $U$ and $\lambda$, the rank of the detection subspace is $\mathcal{U}=\lambda U$.

In addition to the common parameters, such as the spreading factor $N=31$ the number of multipath components $L=4$, used for all figures, there were some specific parameters for each of the figures. In more details, in the context of Fig. 3 and Fig. 4, we assumed that the MIMO SCDMA system employed $V=2$ BS receive antennas and each mobile user employed $U=2$ transmit antennas. The number of users supported was assumed to be $K=5$ for Fig. 3, while $K=10$ for Fig. 4 . Hence, the number of ranks of the signal subspace for Fig. 3

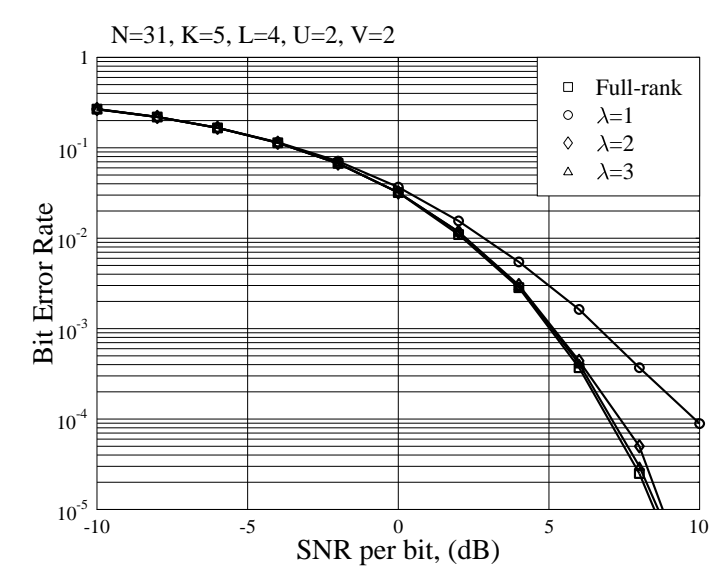

Fig. 3. BER versus SNR per bit performance for the MIMO SCDMA system using TPA-assisted reduced-rank MMSE detection, when communicating over multipath Rayleigh fading channels.

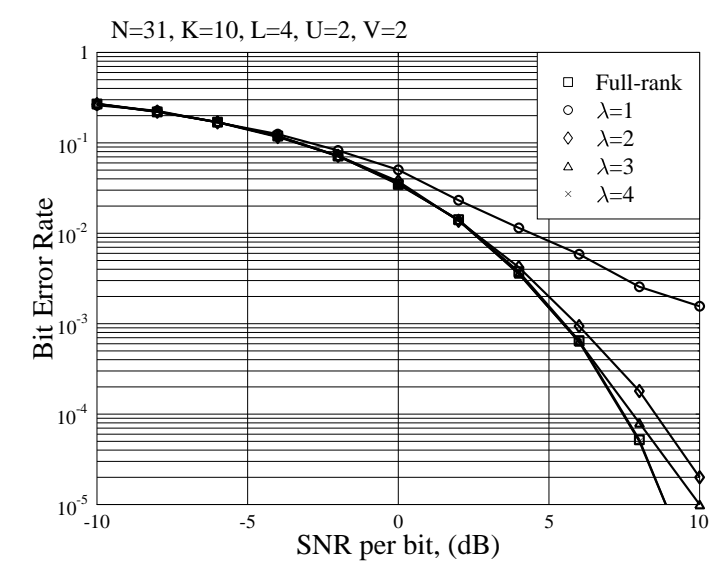

Fig. 4. BER versus SNR per bit performance for the MIMO SCDMA system using TPA-assisted reduced-rank MMSE detection, when communicating over multipath Rayleigh fading channels.

is higher than $2 \times 5=10$, while for Fig. 4 is higher than $2 \times$ $10=20$, since there exists ISI due to multipath propagation. By contrast, in the context of Fig. 5 and Fig. 6, we assumed that the MIMO SCDMA system employed $V=1 \mathrm{BS}$ receive antennas and each mobile user employed $U=4$ transmit antennas. The number of users supported was assumed to be $K=5$ for Fig. 5, while $K=10$ for Fig. 6 . Hence, the number of ranks of the signal subspace for Fig. 5 is higher than $4 \times 5=20$, while for Fig. 6 is higher than $4 \times 10=40$, again, due to the ISI.

From the results in Figs. 3 - 6, we can have the following observations.

- For a given SNR value, the BER performance improves, when increasing the rank, $\mathcal{U}=\lambda U$, of the detection subspace, when the rank of the detection subspace is 


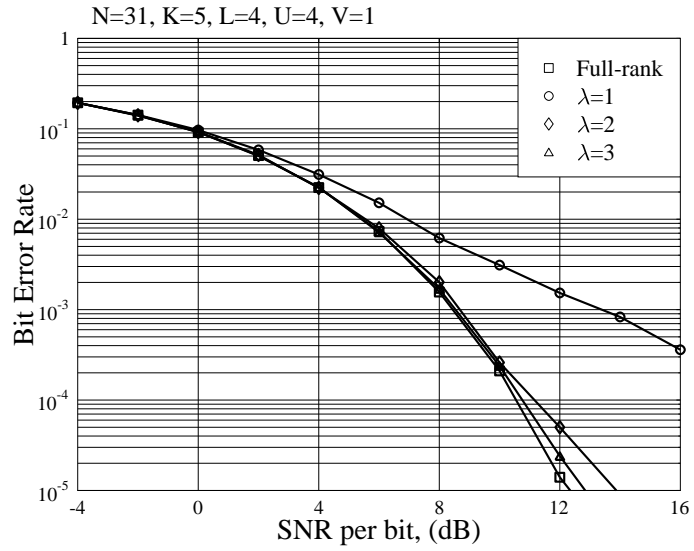

Fig. 5. BER versus SNR per bit performance for the MIMO SCDMA system using TPA-assisted reduced-rank MMSE detection, when communicating over multipath Rayleigh fading channels.

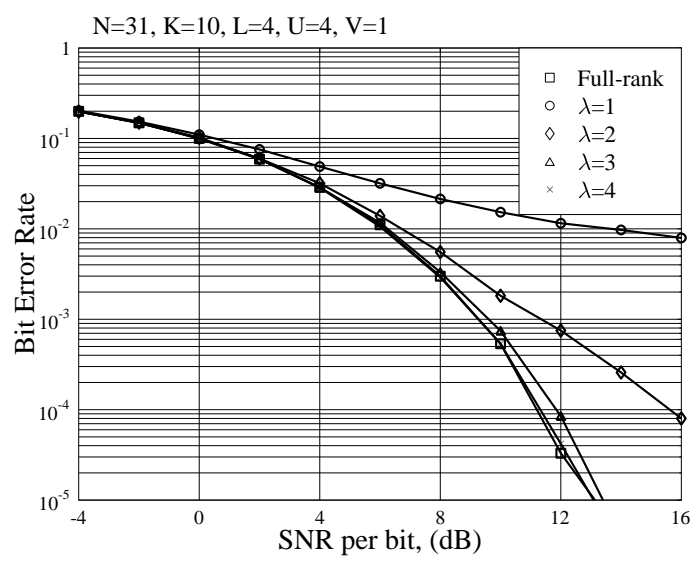

Fig. 6. BER versus SNR per bit performance for the MIMO SCDMA system using TPA-assisted reduced-rank MMSE detection, when communicating over multipath Rayleigh fading channels.

relatively low, such as $\lambda \leq 2$ in all the figures.

- The full-rank BER performance can be achieved, when the rank of the detection subspace is around $\mathcal{U}=\lambda U$ associated with $\lambda=3,4$. This value is significantly lower than the rank of the corresponding signal subspaces, as shown previously in this section.

- No matter what the rank of the signal subspace is, the BER performance of the TPA-assisted reduced-rank MMSE detection converges to the BER performance achieved by the full-rank MMSE detection, provided that the value of $\lambda$ is around $\lambda=3,4$. Specifically, as shown in Fig.6, the full-rank BER performance can be achieved by invoking a detection subspace corresponding to $\lambda=4$, or a detection subspace of rank $\mathcal{U}=16$ that is significantly lower than that of the signal subspace, which is higher than $U \times K=40$. According to the above analysis and the results in Figs. 3 - 6, it can be shown that the $\lambda$ value required to achieve the full-rank BER performance does not scale with the system size determined by the number of users, $K$, as well as the number of receive antennas and the spreading factor.

Note that the TPA-assisted reduced-rank MMSE detection does not depend on the eigen-decomposition of the autocorrelation matrix $\boldsymbol{R}_{y}^{(k)}$.

\section{CONCLUSions}

In conclusions, in this contribution we have investigated the performance of the MIMO SCDMA system combining both space-division and code-division, when communicating over frequency-selective fading channels. Since the full-rank MMSE detection for the MIMO SCDMA is high-complexity due to the long observation vectors invoked, a reduced-rank MMSE detection scheme has been proposed based on the principles of Taylor polynomial approximation (TPA). A range of simulation results have been provided for characterizing the BER performance of the TPA-assisted reduced-rank MMSE detection. From our study and simulation results, it can be shown that the detection complexity in the MIMO SCDMA can be significantly reduced, when the TPA-assisted reduced-rank MMSE detection is employed. The full-rank BER performance can be achieved by invoking a detection subspace with a rank that is significantly lower than that of the signal subspace. Furthermore, it can be shown that the rank required to achieve the full-rank BER performance does not scale with the system size determined by the number of users supported, the number of receive antennas and the spreading factor employed.

\section{ACKNOWLEDGEMENT}

The author would like to acknowledge with thanks the financial assistance from EPSRC of UK.

\section{REFERENCES}

[1] L.-L. Yang, "MIMO assisted space-code-division multiple-access: Linear detectors and performance over multipath fading channels," IEEE J. on Select. Areas in Commun., vol. 24, pp. 121 - 131, Jan. 2006.

[2] S. Verdu, Multiuser Detection. Cambridge University Press, 1998.

[3] S. Moshavi, E. G. Kanterakis, and D. L. Schilling, "Multistage linear receivers for DS-CDMA systems," International Journal of Wireless Information Networks, vol. 3, pp. 1 - 17, January 1996.

[4] B. Hochwald, T. L. Marzetta, and C. B. Papadias, "A transmitter diversity scheme for wideband CDMA systems based on space-time spreading," IEEE J. on Select. Areas in Commun., vol. 19, pp. 48-60, Jan. 2001.

[5] J. G. Proakis, Digital Communications. McGraw Hill, 3rd ed., 1995.

[6] H. L. V. Trees, Optimum Array Processing. Wiley Interscience, 2002.

[7] J. S. Goldstein, I. S. Reed, and L. L. Scharf, "A multistage representation of the Wiener filter based on orthogonal projections," IEEE Trans. on Inf. Theory, vol. 44, pp. 1943 - 2959, Nov. 1998.

[8] M. L. Honig and J. S. Goldstein, "Adaptive reduced-rank interference suppression based on the multistage Wiener filter," IEEE Trans. on Commun., vol. 50, pp. 986 - 994, June 2002.

[9] L.-L. Yang, "Reduced-rank MMSE detection in space-time coded spacedivision multiple-access systems," in IEEE PIMRC'06, IEEE, September 2006 . 